\title{
Study on Air Flow Characteristics in Cylinders of a Four-Valve Engine with Different Lifts of Valves
}

\author{
Shiqiang Zhang ${ }^{*},{ }^{,}$Xianghong Zhang ${ }^{1}$, Huigang Wang ${ }^{1}$, Ruilin Liu ${ }^{2}$, Wuquan Liu ${ }^{2}$, \\ Guangmeng Zhou ${ }^{2}$ and Miao $\mathrm{Chi}^{2}$ \\ ${ }^{I}$ Department of Electro-Mechanical Engineering, Tangshan College, Tangshan 063000, China \\ ${ }^{2}$ Automobile Engineering Department, Military Transportation University, Tianjin 300161, China
}

\begin{abstract}
A 1arge-scale tumble rather than swirl would exist in cylinders in the case of two valves' opening in a fourvalve engine. A strong swirl is generated when one valve is closed, yet the flow capacity is less than that of two valves being opened. In order to change it, different lifts of valves (DLV) present are achieved. In the case of using different lifts of two intake valves, both tumble and swirl would occur in cylinders, and the flow capacity will hardly change. A new method of measuring the rotational speed of the intake swirl -the method of virtual rotational speed of theory has been put forward. Not only does it eliminate the influence of frictional resistance, but also obtain a theoretic rotational speed, which is only produced by intake swirl.
\end{abstract}

Keywords: Different lifts of valves, engine, swirl, virtual rotational speed of theory.

\section{INTRODUCTION}

Air motion in engines has decisive influence on mixing gas and burning process [1]. Organizing favorable gas glowing is a useful way to meet strict standards on emissions and fuel economy. Colossal work has been done, such as increasing the diameter of intake ports, adding number of intake ports, adopting Variable Induction Controlling System (VICS) [2-4], Variable Valve Actuation (VVA) [5-7] and Variable Event Timing Control (VTEC) [8]. Especially, adopting two intake ports increases the ability of circulation, but matching cams make two intake valves move with same lifts, which leads to symmetry of gas flowing. And a largescale tumble rather than swirl would exist in the case of two valves' opening $[9,10]$. To gain a strong swirl, one valve must be closed. However, the flow capacity is less than that of double valves being opened. In order to change it, a new design scheme of matching cam-achieving different lifts of valves (DLV) is present in the patent of invention [11]. So both tumble and swirl would exist in cylinders in the case of using different lifts of two intake valves. Meanwhile the flow capacity will hardly change, the intake port flow characteristics will be improved. In the present work the authors investigate the effects of DLV for swirl and flow capacity.

\section{ACHIEVING DIFFERENT LIFTS OF VALVES}

\subsection{Definition}

Different lifts of valves indicate that lifts of valves are different when two intake valves are moving at the same time. It is relative to the same lifts of valves (SLV) in the engine.

*Address correspondence to this author at the Department of ElectroMechanical Engineering, Tangshan College, Tangshan 063000, China; Tel: +86-0315-2010536; Fax: +86-0315-2010536; E-mail: zsq933@126.com

\subsection{Realization}

The framework of two matching cams is redesigned. The same lifts of cams are considered as the reference cam which is shown in Fig. (1), and change its disposal and profile curve. Four basic schemes are shown in Fig. (2), which make valves to have four sporting forms: According to scheme a, their lifts are different when valves are opening but equal when valves are closing; According to scheme $b$, the lifts are equal when valves are opening but different when valves are closing; According to scheme c, the lifts are different when valves are both opening and closing except for a certain lift in middle of the movement; According to scheme $\mathrm{d}$, the lifts have dissimilarities not only when valves are opening but also when they are closing except for the biggest lift.

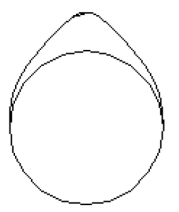

Fig. (1). Reference cam of SLV.

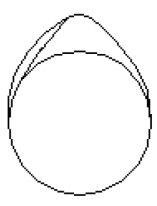

Scheme a

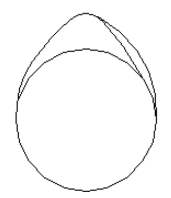

Scheme b

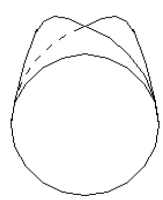

Scheme c

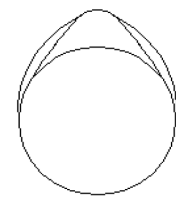

Scheme d
Fig. (2). Four basic schemes of DLV.

\section{CONTRASTING AND ANALYZING}

The better scheme can be found by comparing the ability of circulating, the degree of swirl and tumble. In contrast to the case of the lifts of valves being identical, the airflow is so 
unsteady when engine is running, so that becomes difficult to test the intensity of swirl and tumble directly in the engine. The flow performance of the ports in a cylinder head is tested in a steady-state air rig in Fig. (3).

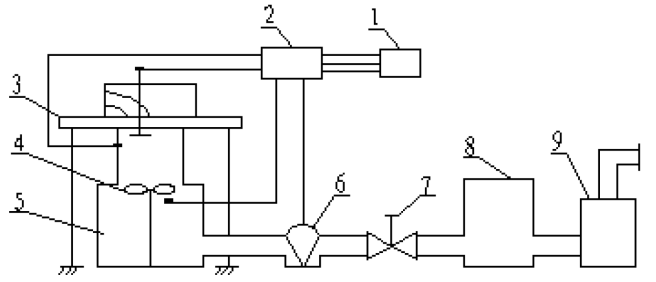

1 computer 2 gathering implement 3 testing bench 4 vane 5 manostat 6 flowmeter 7 adjustor 8 manostat 9 blower

Fig. (3). Sketch of steady flow rig.

\subsection{Estimating Method}

Swirl and tumble are dependent upon many things, including port orientation, chamber masking, and number of valves etc. It is beneficial to estimate air motion by analyzing the bench data in terms of non-dimensional parameters so as to allow comparisons independent of size. Generally, the Ricardo method is adopted to estimate incylinder air motion.

The non-dimensional parameters utilized to describe flow, swirl and tumble conditions are:

Flow Coefficient $C_{F}=\frac{Q}{n \cdot A \cdot V_{0}}$

Average flow Coefficient $\bar{C}_{F}=\frac{1}{\alpha_{2}-\alpha_{1}} \int_{\alpha_{1}}^{\alpha_{2}} C_{F} \cdot d \alpha$

Non-Dimensional Swirl $N_{R}=\frac{\omega_{R} B}{V_{0}}$

Non-Dimensional Tumble $R_{t}=\frac{N_{t(a)} \cdot V_{s}}{30 \cdot Q_{(a)}}$

where:

$Q Q_{(\alpha)}$ are the total volume flow $\left(\mathrm{m}^{3} / \mathrm{sec}\right)$

$n$ is the number of valves open

$A$ is valve seat area $\left(\mathrm{m}^{2}\right)$

$A=\pi D^{2} / 4$

$\alpha_{1} \alpha_{2}$ are crank angle degrees when the valves begin to open and close entirely(rad)

$B$ is the bore $(\mathrm{m})$

$D$ is the inner valve seat diameter $(\mathrm{m})$

$\omega_{R} \quad N_{t(\alpha)}$ are the equivalent swirl velocity(r/min)

$V_{s}$ is the ejecting quantity

$V_{0}$ is the theoretical velocity of the port $(\mathrm{m} / \mathrm{sec})$

$V_{0}=\sqrt{2 \Delta p / \rho}$
$\Delta p$ is the stress drop of the intake port

$\rho$ is the density of the gas at the valve seat $\left(\mathrm{kg} / \mathrm{m}^{3}\right)$

\subsection{Virtual Rotational Speed of Theory}

Steady-state air rig in Fig. (3) has some shortcomings in the assessment test of the intensity of the intake swirl. Intake swirl overcomes frictional resistance to make a vane rotate when the rotational speed of a vane is being tested. The influence of frictional resistance makes measuring result becomes smaller; as a result the residual becomes bigger.

Virtual rotational speed of theory (VRST) can conquer the shortcomings mentioned above. The measuring principle is shown in Fig. (4). Intake swirl is regarded as resistance. The motor drives a vane to rotate when the valve is closed, the direction is opposite to intake swirl. The rotational speed of vane is $\omega_{1}$. Then the valve is opened, intake swirl resisting the vane rotating which makes the speed of the vane to decline, speed of the vane is $\omega_{2}$ at that moment. According to the theorem of kinetic energy, the work of resistance of intake swirl treated as motive power can be obtained. Assuming the vane is derived by them, the theoretical speed of the vane is calculated. Without the impact of frictional resistance, the speed is produced only by intake swirl. In fact it isn't reflected by means of vanes, so it is called "virtual rotational speed of theory", which is marked $\omega_{x}$.

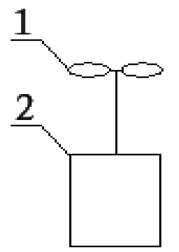

Fig. (4). Driving device.

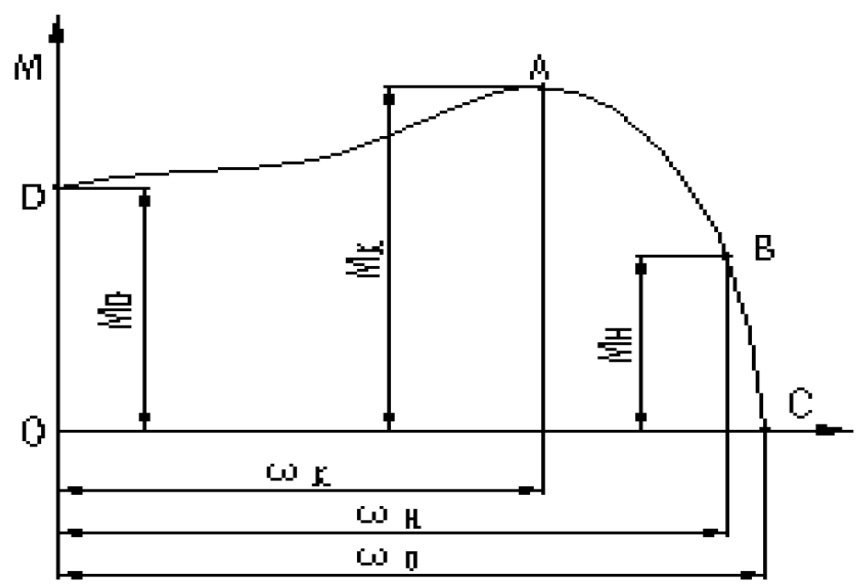

Fig. (5). Mechanical properties of motor.

Mechanical properties of three-phase asynchronous motor are shown in Fig. (5). AD is unstable, and $\mathrm{AC}$ is steady. The parameters are given as follows:

$P_{H}$ is rated power(r/min)

$n_{0}$ is synchronous speed $(\mathrm{r} / \mathrm{min})$ 
$n_{H}$ is rated speed(r/min)

$\lambda=M_{K} / M_{H} \quad M_{K}$ is $\max$ torque(n.m); $M_{H}$ is Rated torque(n.m)

$\lambda_{1}=M_{D} / M_{H} \quad M_{D}$ is starting torque(n.m); $M_{H}$ is Rated torque(n.m)

Hence

$\mathrm{A}\left(\omega_{K}, M_{K}\right), \mathrm{B}\left(\omega_{H}, M_{H}\right), \mathrm{C}\left(\omega_{0}, 0\right), \mathrm{D}\left(0, M_{D}\right)$

Among them

$M_{H}=9550 \frac{P_{H}}{n_{H}}, \omega_{H}=\frac{\pi}{30} n_{H}, \omega_{0}=\frac{\pi}{30} n_{0}$

$M_{K}=\lambda M_{H}, M_{D}=\lambda_{1} M_{H}, \omega_{K} \approx \omega_{0}-\left(\omega_{0}-\omega_{H}\right)\left(\lambda+\sqrt{\lambda^{2}-1}\right)$

$\mathrm{AC}$ is showed by quadratic function

$M=a+b \omega+c \omega^{2}$

Inserting (1)

$\left\{\begin{array}{l}a+b \omega_{0}+c \omega_{0}^{2}=0 \\ a+b \omega_{H}+c \omega_{H}^{2}=M_{H} \\ a+b \omega_{K}+c \omega_{K}^{2}=M_{K}\end{array}\right.$

We obtain

$\left\{\begin{array}{l}a=\omega_{0} \omega_{K} \alpha+\omega_{0} \omega_{H} \beta \\ b=-\left(\omega_{0}+\omega_{K}\right) \alpha-\left(\omega_{0}+\omega_{H}\right) \beta \\ c=\alpha+\beta\end{array}\right.$

In the formula

$\left\{\begin{array}{l}\alpha=\frac{M_{H}}{\left(\omega_{H}-\omega_{0}\right)\left(\omega_{H}-\omega_{K}\right)} \\ \beta=\frac{M_{K}}{\left(\omega_{K}-\omega_{0}\right)\left(\omega_{K}-\omega_{H}\right)}\end{array}\right.$

So $M$ is obtained

According to the theorem of kinetic energy

$\Delta E_{k}=W \quad W=W_{q}-W_{z}$

$W_{q}$ is work of drive torque

$W_{z}$ is work of resistance

Hence

$\frac{1}{2} J \omega_{2}^{2}-\frac{1}{2} J \omega_{1}^{2}=W_{q}-W_{z} W_{q}=\int_{\phi_{1}}^{\phi_{2}} M d \phi$

$M$ is drive torque of motor

$W_{z}$ is work of resistance of intake swirl

$\frac{1}{2} J \omega_{2}^{2}-\frac{1}{2} J \omega_{1}^{2}=W_{q}-W_{z} W_{q}=\int_{\phi_{1}}^{\phi_{2}} M d \phi$
Hence

$W_{z}=W_{q}-\left(\frac{1}{2} J \omega_{2}^{2}-\frac{1}{2} J \omega_{1}^{2}\right)$

Virtual rotational speed of theory

$W_{z}=\frac{1}{2} J \omega_{x}^{2}-0 \quad \omega_{x}=\sqrt{\frac{2 W_{z}}{J}}$

\subsection{Experiment}

Sample is a four-valve gasoline engine. Flow coefficient, non-dimensional swirl and non-dimensional tumble will be obtained based on above formulas.

The result is shown in Fig. (6). The lifts of valves are different when they are opening in scheme a, the balance of swirl has been destroyed. This leads to a big scale swirl, and it changes from weak to strong, and at the end becomes weak again and disappears. Although their lifts are equal when valves are closing, but the inertia of the swirl will occur when valves began to close, the big scale swirl will not disappear immediately. Scheme $b$ describes that the lifts of valves are equal when valves are opening, but their lifts have dissimilarities when valves are closing. There is the big scale swirl in the cylinder during the closing of the valves, and they change from weak to strong, and become weak again, and at last disappear. Scheme $\mathrm{c}$ implies that the lifts of valves are different during valves are opening and closing except a certain lift in the middle of motion, so the big scale swirl will exist all the time except a certain lift in middle of the movement. This is because there is the same lift at the middle of motion, and because of the influence of the inertia of the swirl at the same lift, the big scale swirl will not disappear in a short while, then it will reappear with the emergence of the different lifts. But because intensity of swirl of the intake ports has been transformed, so the direction of the swirl is opposite comparing with previous one. It will disappear gradually with the difference of lifts decreasing. Scheme d makes the lifts of valves have dissimilarities not only during valves are opening but also during they are closing except the biggest lift, the big scale swirl always exists, because the intensity of swirl of intake ports does not transform, so the direction of the swirl is as same as the first one, although there is a same lift in the middle. But because of the influence of the inertia of the swirl at the same lift, the big scale swirl will weaken gradually but still exist; it will strengthen with the increase of the difference of lifts again, and weaken gradually with the decrease of difference of lifts. At last, it will disappear when the valves close completely.

The bigger scale swirl will exist once in Scheme a and scheme b; The bigger scale swirl will exist twice in scheme $\mathrm{c}$, and the difference of lifts in the case of scheme $\mathrm{c}$ is more obvious, the intensity of swirl produced by each intake port tend to be more imbalanced, so the total intensity of the big scale swirl is higher than other cases. Though scheme d produces also bigger scale swirl twice, but because of the limitation of difference of lifts, the imbalanced degree of swirl is lower than that of scheme c, thus the biggest intensity of swirl is less than that of scheme c. In short, scheme c brings better result. 


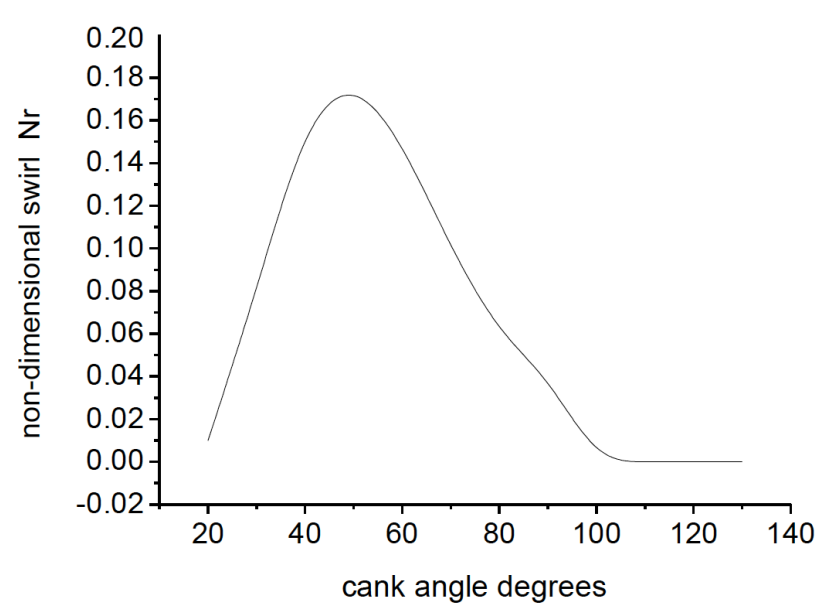

Scheme a

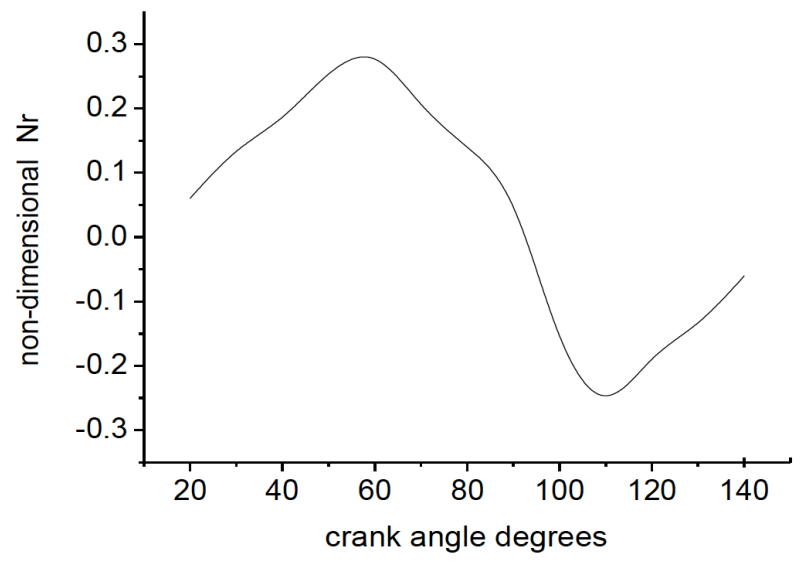

Scheme c

Fig. (6). Non-dimensional swirl $\mathrm{Nr}$ of four schemes.

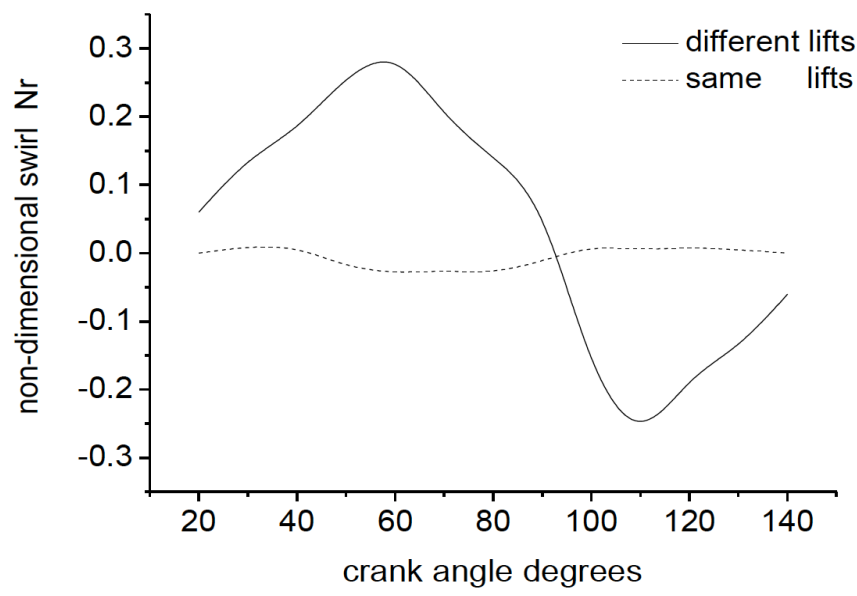

Fig. (7). Non-dimensional swirl $\mathrm{Nr}$ of two conditions.

Fig. (7) shows that the rotating speed of swirl of a traditional four-valve engine is so slow that it cannot be measured steadily, and the direction of swirl often changes. It also shows that there is no big scale swirl in the cylinder, which is attributed to the symmetrical disposal of intake ports and same lifts of the valves that lead to counteract swirl produced by each intake port.
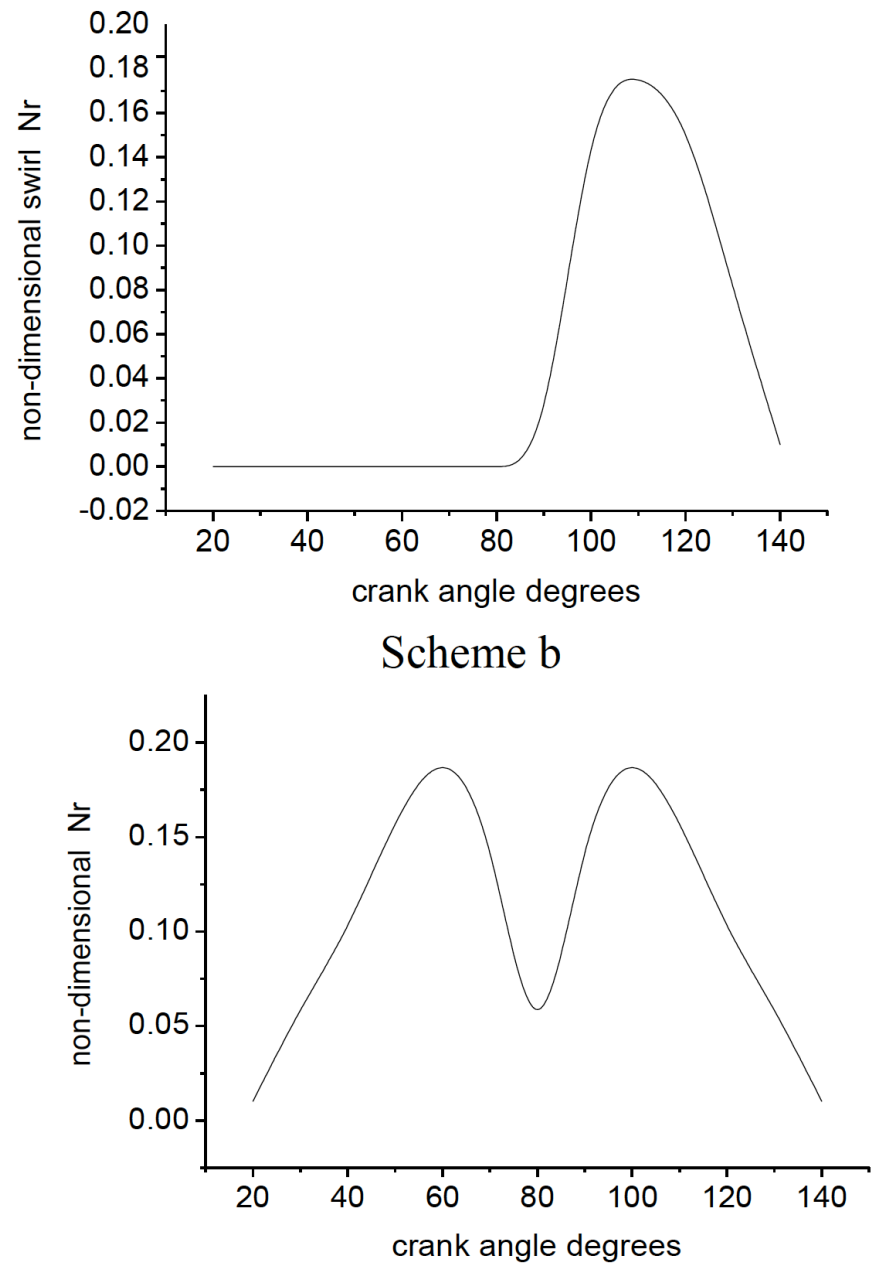

Scheme d

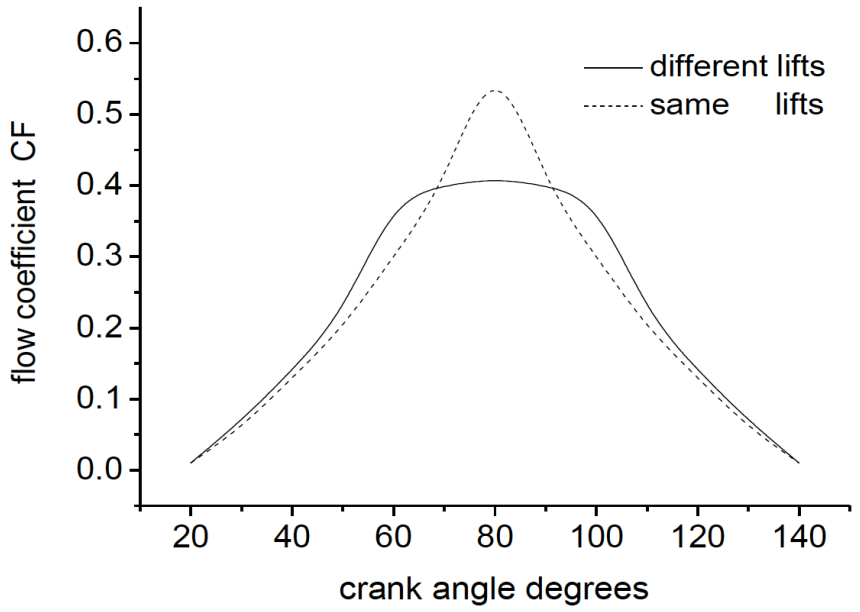

Fig. (8). Flow coefficient CF of two conditions.

Flow coefficient is reduced by 80 degrees in the case of scheme $\mathrm{c}$ than that of same lifts, because two valves in the case of same lifts reach the biggest lift at the same time rather than that of the scheme $\mathrm{c}$, but the average flow coefficient hardly changes in the whole course that is shown in Fig. (8). 


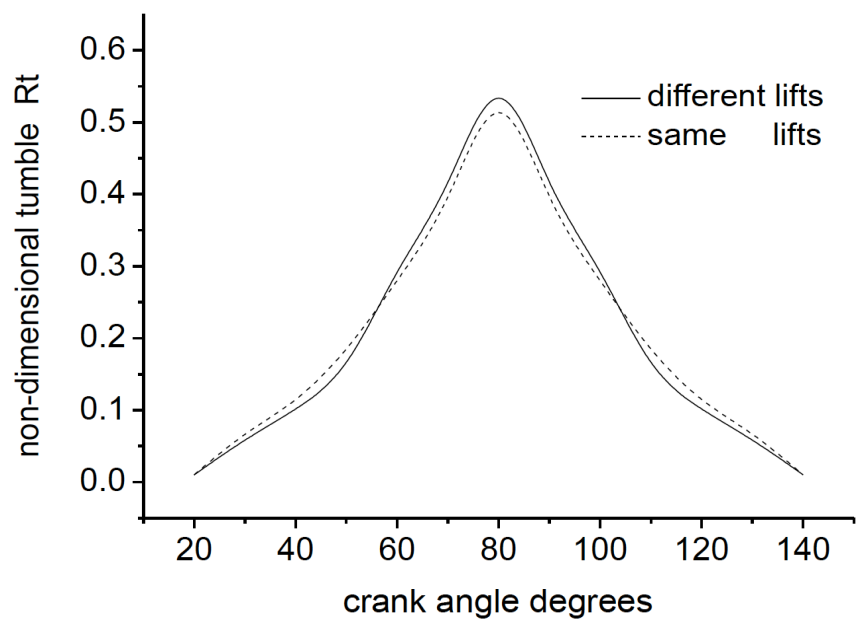

Fig. (9). Non-dimensional tumble Rt of two conditions.

Fig. (9) shows that same question does not happen to tumbles, its intensity will enhance a little by 80 degrees. This is because the sum of lifts at the place in the case of scheme $\mathrm{c}$ is less than that of same lifts, and flow speed of air has increased which causes speed of tumble increased, but the average intensity of tumbles hardly changes.

\section{CONCLUSION}

VRST is based on analyzing the shortcomings in the assessment test of the intensity of the intake swirl in the internal-combustion engine. It is a theoretic rotational speed which is only produced by intake swirl. The intensity of the intake swirl calculated by VRST is more practical.

DLV can conquer that flux is decreased when a valve is closed for swirl and that swirl is lost when two valves are open for flux. It gives attention to the swirl and flux. The largest intensity of tumble is also heightened somehow. Especially, scheme $\mathrm{C}$ improves the mixture of fuel and spread of blaze, it also quickens burning speed remarkably. In addition, a majority of characteristics of the engine can be studied in the DLV and comparisons with the case of SLV can be done. Further discussion and validation on the DLV can be made.

\section{CONFLICT OF INTEREST}

The authors confirm that this article content has no conflict of interest.

\section{ACKNOWLEDGEMENTS}

Thanks for the funds supported by the Special Funds of the National Natural Science Foundation of China (51246010).

\section{REFERENCES}

[1] L. Kihyung, B. Choongsik, and K. Kernyong, "The effects of tumble and swirl flows on flame propagation in a four-valve SI engine ", Applied thermal engineering, vol. 27, pp. 2122-30, 2007.

[2] R. L. Liu, L. Z. Guan, Z. G. Liu, Z. J. Duan, C. L. Song, "Studies on steady measurements of in-cylinder Swirl in a 4-valve gasoline engine", Transactions of Csice, vol. 19, pp. 377- 82, 2001.

[3] R.L. Liu, Z.Y. Liu, J. Gao, X. G. Zhao, C. L. Zhao, K. Ma, "Experimental study on the in-cylinder swirl characteristics of a four-valve gasoline engine with variable inclined swirl system", Chinese internal combustion engine engineering, vol. 27, pp. 2932, 2006 .

[4] P. Kreuter, P. Heuser and R.M. Joachim, "The meta VVH systemA continuously variable valve timing system", SAE Paper, vol. 50, pp. 643-656, SAE 980765, 1998.

[5] J.W. Yi, W. Steve, and M.J. Jeff, "Understanding of intake cam phasing effects on the induction and fuel-air mixing in a DISI engine", SAE Paper, vol. 83, pp. 1947-58, SAE 2004-01-1947, 2004.

[6] D. Richard Stone, and K. Eric, "Variable valve actuation mechanisms and the potential for their application", SAE Paper vol. 79, pp. 956-63, SAE 890673, 1989.

[7] T. Dresner, and P. Barkan,"A review and classification of variable valve mechanisms", SAE Paper, vol. 65, pp. 1237-49, SAE 890674, 1989.

[8] K. Horie, K. Nishizawa, and T. Ogawa, "The development of a high fuel economy and high performance four-valve lean burn engine", SAE Paper, vol. 35, pp. 507-20, SAE 920455,1992.

[9] P. Kreuter, "Strategies to improve SI-engine performance by means of variable intake lift, timing and duration", SAE Paper, vol. 37, pp. 1265-73, SAE 920449, 1992.

[10] J. Wang, D.X. Liu, and S.L. Liu, "Study of steady measurements on intake port flow characteristics in a four-valve gasoline engine ", Transactions of CSICE, vol. 2, pp. 182-6, 2004(in Chinese).

[11] S.Q. Zhang, "The device and some methods Achieving different lifts of intake valves in an internal-combustion engine ", China Patent ZL200510013859.7, June 17, 2005. 